\title{
Variables explicativas de los flujos de compra de alimentos en las Regiones VIII, IX y X de Chile
}

\author{
Berta L. Schnettler y Alfonso Rebollo \\ 'Departamento de Producción Agropecuaria, Universidad de La Frontera. Casilla 54-D, Temuco, Chile. \\ ${ }^{2}$ Departamento de Financiación e Investigación Comercial, Universidad Autónoma de Madrid. España.
}

\begin{abstract}
B. L. Schnettler, and A. Rebollo. 2007. Explanatory variables of purchase flows of food in IX Region, Chile. Cien Inv. Agr. 34(2):91-102. The unequal distribution of commerce in the region displaces purchase flows from the smaller centers to those with greater commercial facilities, thus causing the re-routing of commercial expenditure. To determine the effect of attraction and restriction variables on the purchase flows of food in the VIII ${ }^{\text {th }}$, IX ${ }^{\text {th }}$ and $\mathrm{X}^{\text {th }}$ Regions of Chile, the traditional gravitational model was used. Purchasing center attributes were population, monthly domestic income, number of business licenses and the commercial surface of establishments selling food. The time and distance of displacement were used as restriction variables. The consumers that travel outside their district of origin to buy food choose those interurban commercial centers with the greatest population where the homes with the greatest income are concentrated and whose commercial supply is greater when measured in the number of businesses or the amount of retail surface. Consumer income is not significant to interurban purchase flow as the small number of commercial facilities in the districts of origin force the consumers to travel.
\end{abstract}

Key words: Commercial flows, commercial gravitation, trade areas.

\section{Introducción}

En las áreas comerciales, los habitantes de los núcleos de población gravitan sobre un centro urbano dotado de un equipamiento comercial bien abastecido. Así, parte del gasto comercializable de los hogares se evade entre localidades, dando cuenta de la existencia de flujos comerciales. Estos se producen por la desigual distribución del equipamiento comercial en el territorio, que responde a la heterogénea distribución de la demanda y las necesidades de rentabilización de las empresas minoristas.

El estudio de los flujos de compra y de la conformación de áreas de comercio se puede hacer mediante modelos analíticos o métodos empíricos. Estos últimos se realizan sobre

Recibido 25 de octubre 2006. Aceptado 26 de marzo 2007.

'Dirigir correspondencia a Berta Schnettler: bschnett@ufro.cl la base de encuestas acerca de los hábitos de compra y la importancia de los flujos de gasto. Esto se dirige a los consumidores o conocedores de los hábitos de compra de los habitantes de una localidad (De Juan, 1998; Casares y Rebollo, 2000). Los métodos analíticos por medio de modelos de comportamiento del consumidor y la estimación de sus parámetros, determinan la amplitud geográfica y el grado de atracción comercial que ejerce un centro de compras. El modelo propuesto por Huff (1964), el más utilizado entre quienes usan información revelada por el consumidor, establece un modelo de atracción comercial desagregado probabilista. Así, surgen los modelos estocásticos cuya generalización origina los modelos de interacción espacial (Fotheringham y O’Kelly, 1989; Gil et al., 1997; Más, 1996; y O’Kelly, 1999). Estos últimos modelos se han usado en Europa y EUA para explicar flujos de compra de productos de compra normal. Sin embargo, no se han empleado para productos de conveniencia, 
como productos para la alimentación. Estos representan aproximadamente $27 \%$ del gasto de los hogares en Chile (INE, 2001).

Al elegir un centro de compras, la conducta del consumidor está en función de variables de atracción y de disuasión. Estas fomentan y dificultan los desplazamientos de los compradores. La variable de restricción se relaciona con la distancia que debe recorrer el consumidor o el tiempo del desplazamiento de compra. Las variables de atracción se relacionan con el tamaño del comercio. La medición de la atracción minorista se basa en la utilización los centros de compra de la población de y está en función creciente al del tamaño físico (superficie comercial) del establecimiento minorista. Cuanto mayor sea el tamaño de un establecimiento o aglomeración de establecimientos, mayor es la variedad de surtido. De este modo, el consumidor obtendrá los artículos en dicho centro y se reduce el riesgo de realizar un desplazamiento de compra infructuoso debido a la mayor variedad de oferta existente en los grandes centros ( $\mathrm{La}$ Forge et al., 1984; Medina, 1997).

Según Dallaert y Arentze (1998), los consumidores prefieren combinar compras de diferentes tipos de productos en sus viajes, reduciendo así, el número de desplazamientos. Según Schnettler (2003), esto explicaría que una parte de los alimentos se adquiera fuera de la comuna de residencia, situación demostrada al estudiar los flujos comerciales de estos productos en comunas de las Regiones VIII, IX y $\mathrm{X}$ de Chile. La actividad de compra implica desplazamiento con un costo en tiempo y dinero. Una mayor distancia entre el establecimiento comercial y el consumidor incide negativamente en la atracción ejercida sobre éste. La distancia es una variable explicativa del patronaje de establecimientos de venta de alimentos (Hortman et al., 1990). $\mathrm{Al}$ aumentar esta variable disminuye la utilidad del consumidor (Marjanen, 1997; Raijas 1997; Orpana y Lampinen, 2003).

El mayor ingreso de los consumidores se relaciona de forma positiva con la compra interurbana (Hawes y Lumpkin, 1984; Bromley y Thomas, 1995; Sullivan y Savitt, 1997). Según
Serrano (1990), a medida que crece el ingreso los desplazamientos de compra aumentan más que proporcionalmente, produciéndose una transferencia del gasto desde las pequeñas localidades a las grandes ciudades. Matthews (1992) determinó que los habitantes de zonas rurales de bajos ingresos poseen un limitado acceso a los supermercados donde obtienen productos alimenticios más baratos.

En los últimos años se ha observado, en el sector alimentario en particular, una fuerte penetración de las cadenas de supermercados e hipermercados en la distribución al detalle en general. Según cifras de la Asociación Gremial de Supermercados de Chile (2001), el sector supermercados varió en promedio desde $49 \%$ del comercio detallista en 1994 a 61\% en 2001. Esta expansión se realizó a costa del sector minorista tradicional, mermando su presencia en la distribución alimentaria.

Esta situación caracteriza también a las ciudades de la zona centro-sur de Chile, donde existen tres importantes centros de compra en la VIII Región (Concepción, Chillán y Los Angeles), un centro principal en la IX Región (Temuco) $\mathrm{y}$ tres centros de atracción comercial en la $\mathrm{X}$ Región (Puerto Montt, Osorno y Valdivia), los que presumiblemente atraen la mayor parte de los flujos evadidos desde las comunas de menor tamaño. En la IX Región, destacan además como centros de atracción comercial secundarios las ciudades de Angol y Villarrica. Por lo tanto, el objetivo del presente estudio fue determinar el efecto de variables de atracción y de restricción en los flujos comerciales de alimentos en comunas de las Regiones VIII, IX y X de Chile.

\section{Materiales y métodos}

Para determinar los factores que explican los flujos comerciales de alimentos entre comunas de las Regiones VIII, IX y X de Chile, se utilizó el modelo de interacción espacial sin restricciones (Fotheringham y O'Kelly, 1989):

$$
F_{i j}=k \cdot V_{i}^{-\beta 1} \cdot W_{j}^{\beta 2} \cdot c_{i j}^{-\beta 3}
$$

donde: $\mathrm{F}_{\mathrm{ij}}$ son los flujos entre el origen i y el 
destino $\mathrm{j} ; \mathrm{V}_{\mathrm{i}}$ son las características del origen $\mathrm{i}$ : $\mathrm{W}_{\mathrm{j}}$ : son las características del destino $\mathrm{j}$; $\mathrm{c}_{\mathrm{ij}}$ es la variable de separación espacial desde el origen $\mathrm{i}$ al destino j; $\mathrm{k}$ corresponde a la constante gravitacional; $\beta_{1}, \beta_{2}, \beta_{3}$, son parámetros del modelo.

Dado que el modelo es potencial multiplicativo, se estimó mediante transformaciones logarítmicas con la forma funcional:

$$
\ln F_{i j}=\ln k-\beta_{1} \ln V_{i}+\beta_{2} \ln W_{j}-\beta_{3} \ln c_{i j}
$$

Los flujos comerciales, medidos como porcentaje de gasto realizado fuera de la comuna de residencia, correspondieron a los obtenidos por Schnettler (2003). Estos se obtuvieron mediante encuestas a informantes calificados de 47 comunas de las Regiones VIII, IX y X, considerando el porcentaje de gasto evadido hacia la comuna de primer o segundo destino elegido para realizar las compras fuera de la comuna de residencia (Cuadros 1 y 2). Como variable de restricción se utilizó la distancia y el tiempo de desplazamiento. Como medida de la atracción comercial se utilizaron cuatro variables en forma sucesiva: 1. Población (número de habitantes totales) de la comuna de origen y destino (INE, 2003), 2. Ingreso monetario mensual de los hogares (MIDEPLAN, 2000), 3. Número de patentes comerciales de tiendas tradicionales de venta de alimentos y supermercados, y 4. Superficie comercial. Se estimaron cinco especificaciones del modelo:

$$
\begin{aligned}
& F_{i j}=k \cdot P_{i}^{-\beta 1} \cdot P_{j}^{\beta 2} \cdot d_{i j}^{-\beta 3} \\
& F_{i j}=k \cdot P_{i}^{-\beta 1} \cdot P_{j}^{\beta 2} \cdot t_{i j}^{-\beta 3} \\
& F_{i j}=k \cdot M_{i}^{\beta 1} \cdot M_{j}^{\beta 2} \cdot t_{i j}^{-\beta 3} \\
& F_{i j}=k \cdot L_{i}^{-\beta 1} \cdot L_{j}^{\beta 2} \cdot t_{i j}^{-\beta 3} \\
& F_{i j}=k \cdot S_{i}^{-\beta 1} \cdot S_{j}^{\beta 2} \cdot t_{i j}^{-\beta 3}
\end{aligned}
$$

donde: $\mathrm{P}_{\mathrm{i}}$ es la población del origen $\mathrm{i} ; \mathrm{P}_{\mathrm{j}}$ es la población del destino $\mathrm{j} ; \mathrm{IM}_{\mathrm{i}}$ es el ingreso monetario (\$) mensual de los hogares del origen $\mathrm{i}$; $\mathrm{IM}_{\mathrm{j}}$ es el ingreso monetario (\$) mensual de los hogares del destino $\mathrm{j} ; \mathrm{L}_{\mathrm{i}}$ es el número de patentes comerciales del origen $\mathrm{i} ; \mathrm{L}_{\mathrm{j}}$ es el número de patentes comerciales del destino $\mathrm{j}$; $\mathrm{S}_{\mathrm{i}}$, es la superficie comercial $\left(\mathrm{m}^{2}\right)$ del origen $\mathrm{i}$; $\mathrm{S}_{\mathrm{j}}$ es la superficie comercial $\left(\mathrm{m}^{2}\right)$ del destino $\mathrm{j}$; $\mathrm{d}_{\mathrm{ij}}$ es la distancia $(\mathrm{km})$ desde el origen $\mathrm{i}$ al destino $\mathrm{j}$; $\mathrm{t}_{\mathrm{ij}}$ es el tiempo de desplazamiento (min) desde el origen $\mathrm{i}$ al destino $\mathrm{j}$.

Los signos de las variables utilizadas implican que cabe esperar que cuanto menor sea la oferta minorista de una determinada localidad, mayor será la propensión a comprar fuera por parte de los habitantes de dicha comuna. Es decir, existe una relación inversa entre los flujos de gasto y la oferta minorista de las comunas. Además, se debe esperar una relación claramente positiva entre los flujos de gasto y el tamaño de los centros de compra interurbanos. Debido a que la distancia constituye una variable de restricción de los flujos de gasto, se debe esperar una relación inversa entre los flujos y esta variable. Lo mismo sucede con el tiempo de desplazamiento. Finalmente, se espera que los hogares de las comunas con mayor nivel de renta presenten una mayor predisposición a comprar fuera de su comuna de residencia; es decir, que se produzca una relación positiva entre el ingreso monetario de los hogares y la cuantía de los flujos de gasto evadidos.

Para la estimación de las especificaciones que utilizan las poblaciones y el ingreso monetario de las comunas, se utilizaron los flujos comerciales al primer y segundo destino de compras de la totalidad de las comunas, totalizando 75 flujos observados (Cuadros 1 y 2). En las especificaciones que usan el número de licencias comerciales y la superficie de ventas, sólo se consideraron los flujos de las comunas que conformaron el área comercial de Temuco (Cuadro 1). Esto debido a que fue posible disponer de la información de las variables de atracción utilizadas sólo para este grupo (49 flujos observados). Las especificaciones (5), (6) y (7) se estimaron utilizando el tiempo de desplazamiento, debido a que se logró un mejor ajuste con esta variable de restricción. La estimación del modelo se realizó mediante análisis de regresión múltiple con el método 
Cuadro 1. Flujos comerciales en productos de alimentación determinados para comunas del área comercial de Temuco, Chile. Table 1. Commercial flows in food products for the trade area of the districts of Temuco, Chile.

\begin{tabular}{|c|c|c|c|c|c|c|}
\hline Comunas & $\begin{array}{c}\text { Compra dentro }^{1} \\
\%\end{array}$ & $\begin{array}{c}\text { Compra fuera }^{1} \\
\%\end{array}$ & Primera comuna ${ }^{2}$ & $\%$ & Segunda comuna ${ }^{2}$ & $\%$ \\
\hline Carahue & 80 & 20 & Temuco & 14 & Imperial & 6 \\
\hline Cunco & 30 & 70 & Temuco & 70 & & \\
\hline Curarrehue & 80 & 20 & Pucón & 20 & & \\
\hline Freire & 20 & 80 & Temuco & 80 & & \\
\hline Galvarino & 50 & 50 & Temuco & 40 & Traiguén & 10 \\
\hline Gorbea & 80 & 20 & Temuco & 10 & Pitrufquén & 10 \\
\hline Lautaro & 70 & 30 & Temuco & 30 & & \\
\hline Loncoche & 40 & 60 & Temuco & 40 & Villarrica & 20 \\
\hline Melipeuco & 70 & 30 & Temuco & 20 & Cunco & 10 \\
\hline Imperial & 55 & 45 & Temuco & 45 & & \\
\hline Padre Las Casas & 35 & 65 & Temuco & 65 & & \\
\hline Perquenco & 60 & 40 & Temuco & 38 & Victoria & 2 \\
\hline Pitrufquén & 40 & 60 & Temuco & 40 & Villarrica & 20 \\
\hline Pucón & 80 & 20 & Temuco & 15 & Villarrica & 5 \\
\hline Saavedra & 90 & 10 & Carahue & 10 & & \\
\hline Teodoro Schmidt & 60 & 40 & Temuco & 30 & Toltén & 10 \\
\hline Toltén & 80 & 20 & Temuco & 20 & & \\
\hline Vilcún & 75 & 25 & Temuco & 20 & Lautaro & 5 \\
\hline Villarrica & 90 & 10 & Temuco & 10 & & \\
\hline Collipulli & 95 & 5 & Temuco & 3 & Los Angeles & 2 \\
\hline Curacautín & 70 & 30 & Temuco & 27 & Victoria & 3 \\
\hline Ercilla & 50 & 50 & Collipulli & 35 & Victoria & 15 \\
\hline Lonquimay & 80 & 20 & Temuco & 20 & & \\
\hline Los Sauces & 20 & 80 & Angol & 60 & Temuco & 20 \\
\hline Lumaco & 90 & 10 & Traiguén & 10 & & \\
\hline Purén & 95 & 5 & Temuco & 3 & Angol & 2 \\
\hline Traiguén & 90 & 10 & Temuco & 10 & & \\
\hline Victoria & 80 & 20 & Temuco & 20 & & \\
\hline Lanco & 35 & 65 & Valdivia & 40 & Temuco & 25 \\
\hline Panguipulli & 80 & 20 & Temuco & 12 & Valdivia & 8 \\
\hline Tirúa & 80 & 20 & Cañete & 17 & Carahue & 3 \\
\hline
\end{tabular}

${ }^{1}$ Compras realizadas dentro o fuera de la comuna de origen del consumidor.

${ }^{2}$ Primera y segunda comuna preferida para la compra de alimentos fuera de la comuna de origen.

${ }^{1}$ Purchased made inside or outside of the district where consumer were located.

${ }^{2}$ First and second districts preferred to purchase foods outside of the district of origin.

de mínimos cuadrados ordinarios, empleando el software SPSS (Statistical Package for the Social Sciences para Windows. 2001. Versión 11.0. SPSS Inc. Chicago. USA).

El listado de patentes comerciales de las comunas del área comercial de Temuco se obtuvo mediante solicitud directa a cada municipio. Para calcular la superficie comercial de los establecimientos de venta de alimentos se realizó una encuesta aplicada a una muestra representativa de los dueños de los establecimientos (169 tiendas tradicionales de alimentación de la comuna de Temuco y 268 comercios de las comunas de su área comercial, de un universo de 1.120 y 4.063 patentes; 20 supermercados de Temuco y 48 supermercados de las restantes comunas de un universo de 130 y 492). Se utilizó un cuestionario acerca de la superficie total y la superficie dedicada a la venta en cada establecimiento. La muestra se obtuvo utilizando un muestreo aleatorio estratificado, variable dicotómica, 95\% de confianza y $5 \%$ de error de estimación, con dispersión máxima (Scheaffer et al., 1996). Las encuestas se realizaron entre julio de 2003 y febrero de 2004. La superficie total y de ventas se obtuvo por multiplicación de la media de las encuestas realizadas según la muestra y el universo de patentes.

Para determinar la relación entre población 
Cuadro 2. Comunas de la VIII y X Región de Chile sin atracción comercial por parte de Temuco para la compra de alimentos. Table 2. Districts of the VIIIth and Xth Regions of Chile, without being attracted commercially by Temuco to purchase food.

\begin{tabular}{|c|c|c|c|c|c|c|}
\hline Comunas & $\begin{array}{c}\text { Compra dentro }^{1} \\
\%\end{array}$ & $\begin{array}{c}\text { Compra fuera }^{1} \\
\%\end{array}$ & Primera comuna ${ }^{2}$ & $\%$ & Segunda comuna ${ }^{2}$ & $\%$ \\
\hline Los Alamos & 97 & 3 & Cañete & 2 & Concepción & 1 \\
\hline Cañete & 90 & 10 & Concepción & 8 & Los Alamos & 2 \\
\hline Contulmo & 30 & 70 & Cañete & 42 & Concepción & 28 \\
\hline Curanilahue & 60 & 40 & Concepción & 32 & Arauco & 8 \\
\hline Laja & 30 & 70 & Los Angeles & 49 & Concepción & 21 \\
\hline Nacimiento & 50 & 50 & Los Angeles & 50 & Concepción & \\
\hline Negrete & 20 & 80 & Los Angeles & 60 & Nacimiento & 20 \\
\hline Quilaco & 10 & 90 & Los Angeles & 80 & Concepción & 10 \\
\hline San Rosendo & 35 & 65 & Concepción & 39 & Los Angeles & 26 \\
\hline Sta. Bárbara & 30 & 70 & Los Angeles & 70 & & \\
\hline Yumbel & 60 & 40 & Concepción & 20 & Los Angeles & 20 \\
\hline Corral & 50 & 50 & Valdivia & 50 & & \\
\hline Futrono & 60 & 40 & Valdivia & 35 & Paillaco & 5 \\
\hline La Unión & 80 & 20 & Osorno & 20 & & \\
\hline Lago Ranco & 40 & 60 & Osorno & 60 & & \\
\hline Máfil & 30 & 70 & Valdivia & 70 & & \\
\hline
\end{tabular}

${ }^{1}$ Compras realizadas dentro o fuera de la comuna de origen del consumidor.

${ }^{2}$ Primera y segunda comuna preferida para la compra fuera de alimentos.

'Purchased made inside or outside of the district where consumer were located.

${ }^{2}$ First and second districts preferred to purchase foods outside of the district of origin.

y aglomeración del comercio, las superficies comerciales obtenidas mediante la encuesta a establecimientos comerciales de venta de alimentos, paralelamente, permitieron determinar los parámetros del siguiente modelo:

$$
P_{j}=a+b S_{j}
$$

donde: $S_{j}$ es la superficie comercial de venta de alimentos de una determinada localidad; $\mathrm{P}_{\mathrm{j}}$ es la población de una determinada localidad; a y b son los parámetros del modelo.

\section{Resultados}

\section{Superficie comercial de productos de alimentación}

El número de patentes y superficie de venta correspondiente a tiendas tradicionales de alimentación y supermercados de las comunas del área comercial de Temuco tuvo, en total, $132.284 \mathrm{~m}^{2}$ de tiendas tradicionales y $166.204 \mathrm{~m}^{2}$ de supermercados (Cuadro 3). De la superficie de establecimientos tradicionales de venta de alimentos 20,2\% se concentró en Temuco, mientras que Villarrica y Panguipullituvo entre 6 y $9 \%$. Temuco concentró aproximadamente $26 \%$ de la superficie para supermercados; mientras que las comunas de Lautaro, Traiguén, San José de la Mariquina, Angol y Pitrufquén tuvieron poco más de $5 \%$ cada una. La mayor proporción de superficie de supermercados se encontró en Temuco, pero las mayores superficies promedio por habitante correspondieron a Lanco, San José de La Mariquina, Traiguén, Pitrufquén y Lautaro, comunas donde probablemente existió un uso ineficiente del espacio comercial.

Se debe destacar que la información entregada por las Municipalidades de Ercilla, Puerto Saavedra y Victoria no registró patentes de supermercados. Por lo cual la superficie correspondiente a supermercados fue nula, lo que en el caso de las dos primeras comunas resultó congruente con la escasa población (menos de 15.000 habitantes cada una) que habitan en dichas comunas. Por el contrario, no fue así en Victoria que constituye la sexta comuna en concentración poblacional de la IX Región, después de Temuco, Padre Las Casas, Angol, Villarrica y Nueva Imperial, con una población de 33.501 habitantes en 2002 (INE, 2003). Esta situación se podría deber a la forma de registrar las patentes comerciales, situación que también explicaría la baja cantidad de patentes y superficie comercial de supermercados en Pucón. 
Cuadro 3. Patentes y superficie comercial de alimentos en el área comercial de Temuco, Chile.

Table 3. Business licenses and commercial surface of food products in the Temuco's trade area.

\begin{tabular}{|c|c|c|c|c|}
\hline \multirow[t]{2}{*}{ Comunas } & \multicolumn{2}{|c|}{ Tiendas tradicionales } & \multicolumn{2}{|c|}{ Supermercados } \\
\hline & $\begin{array}{c}\text { Patentes } \\
\text { no. }\end{array}$ & $\begin{array}{c}\text { Superficie } \\
\mathrm{m}^{2}\end{array}$ & $\begin{array}{c}\text { Patentes } \\
\text { no. }\end{array}$ & $\begin{array}{c}\text { Superficie } \\
\mathrm{m}^{2}\end{array}$ \\
\hline \multicolumn{5}{|c|}{ Provincia de Arauco: } \\
\hline Cañete & 225 & $6.277,5$ & 29 & $8.047,5$ \\
\hline Contulmo & 37 & 370,0 & 5 & 350,0 \\
\hline Tirúa & 39 & 780,0 & 5 & 350,0 \\
\hline \multicolumn{5}{|c|}{ Provincia de Malleco: } \\
\hline Angol & 314 & $5.149,6$ & 43 & $10.719,9$ \\
\hline Collipulli & 137 & $5.178,6$ & 24 & $7.860,0$ \\
\hline Curacautín & 131 & $5.226,9$ & 23 & $1.782,5$ \\
\hline Ercilla & 37 & 714,1 & 0 & 0 \\
\hline Lonquimay & 91 & $3.166,8$ & 13 & $2.795,0$ \\
\hline Los Sauces & 56 & 952,0 & 3 & 360,0 \\
\hline Lumaco & 59 & 814,2 & 6 & 336,0 \\
\hline Purén & 79 & $2.291,0$ & 11 & $2.376,0$ \\
\hline Renaico & 78 & $1.887,6$ & 12 & 900,0 \\
\hline Traiguén & 145 & $3.277,0$ & 24 & $11.280,0$ \\
\hline Victoria & 105 & $1.995,0$ & 0 & 0 \\
\hline \multicolumn{5}{|c|}{ Provincia de Cautín: } \\
\hline Temuco & 1.120 & $26.768,0$ & 130 & $43.069,0$ \\
\hline Carahue & 163 & $3.634,9$ & 8 & 560,0 \\
\hline Cunco & 154 & $3.726,8$ & 13 & 910,0 \\
\hline Curarrehue & 61 & $1.970,3$ & 3 & 540,0 \\
\hline Freire & 127 & $3.225,8$ & 3 & $1.200,0$ \\
\hline Galvarino & 63 & $1.512,0$ & 8 & 640,0 \\
\hline Gorbea & 108 & $2.452,7$ & 18 & $1.620,0$ \\
\hline Lautaro & 188 & $4.211,2$ & 29 & $13.775,0$ \\
\hline Loncoche & 181 & $4.796,5$ & 11 & $4.400,0$ \\
\hline Melipeuco & 31 & 775,0 & 6 & 576,0 \\
\hline Imperial & 147 & $3.219,3$ & 10 & $6.400,0$ \\
\hline Padre Las Casas & 241 & $3.398,1$ & 30 & $2.940,0$ \\
\hline Perquenco & 22 & 770,0 & 6 & 420,0 \\
\hline Pitrufquén & 132 & $2.679,6$ & 29 & $9.715,0$ \\
\hline Pucón & 17 & 408,0 & 1 & 16,0 \\
\hline Saavedra & 38 & $2.686,6$ & 0 & 0 \\
\hline Teodoro Schmidt & 72 & $1.656,0$ & 10 & $2.000,0$ \\
\hline Toltén & 76 & $1.976,0$ & 2 & 224,0 \\
\hline Vilcún & 43 & 903,0 & 9 & $6.048,0$ \\
\hline Villarrica & 261 & $11.510,1$ & 1 & $1.100,0$ \\
\hline \multicolumn{5}{|c|}{ Provincia de Valdivia: } \\
\hline Lanco & 105 & $2.173,5$ & 28 & $9.870,0$ \\
\hline Mariquina & 75 & $1.087,5$ & 35 & $10.780,0$ \\
\hline Panguipulli & 225 & $8.662,5$ & 34 & $2.244,0$ \\
\hline
\end{tabular}

Inicialmente, la literatura relacionada con los estudios sobre distribución comercial midieron la atracción comercial de un determinado centro de compras interurbano de acuerdo con su nivel poblacional. Posteriormente, y superando las limitaciones que supone usar la población como variable de atracción se ha utilizado el tamaño del centro comercial en términos de la superficie $\left(\mathrm{m}^{2}\right)$ dedicada al comercio. Sin embargo, es posible esperar que exista una relación directa entre ambas medidas de atracción, por cuanto el equipamiento minorista se agrupa en las localidades de mayor potencial de demanda relativa. Como se observa en 
Cuadro 4. Ecuaciones de regresión entre la población y la superficie comercial de las comunas del área comercial de Temuco, Chile.

Table 4. Regression equations between population and commercial surface of the districts of Temuco's trade area.

\begin{tabular}{|c|c|c|c|c|}
\hline Grupo de bienes & Ecuación de regresión & t-student a & t-student $\mathrm{Sj}$ & $\mathrm{R}^{2}, \%$ \\
\hline $\begin{array}{l}\text { Tiendas tradicionales } \\
\text { de alimentación }\end{array}$ & $\mathrm{Pj}=-108,851+7,488 \mathrm{Sj}$ & $-0,040$ & 60,344 & 99,0 \\
\hline Supermercados & $\mathrm{Pj}=1.148,6+5,888 \mathrm{Sj}$ & 0,284 & 40,583 & 97,9 \\
\hline
\end{tabular}

el Cuadro 4, el signo positivo del parámetro "b" correspondiente a la superficie comercial de tiendas tradicionales de alimentación y supermercados y los elevados coeficientes de determinación obtenidos, permiten afirmar que los establecimientos minoristas de venta de alimentos se localizan en función del nivel de demanda existente en el área comercial de Temuco (Cuadro 4). Esto permite inferir que para el caso del área comercial en estudio, la población es un indicador válido de la existencia de comercio tradicional de venta de alimentos y de supermercados en un determinado centro urbano.

\section{Modelación de los flujos comerciales de productos de alimentación}

Según lo esperado, se obtuvieron coeficientes negativos para las variables población, número de patentes comerciales y superficie comercial correspondientes a la comuna de origen del consumidor (Cuadro 5). Por esto es posible indicar que la insuficiente oferta minorista de alimentos provoca desplazamientos hacia centros de compra interurbanos de mayor tamaño, lo que se relaciona con la menor variedad y mayores precios que presenta la oferta de alimentos en localidades de menor

Cuadro 5. Estimación del modelo de gravitación comercial en productos de alimentación, utilizando diferentes variables de atracción y de restricción en el sur de Chile.

Table 5. Estimation of the commercial gravitational model on food products in the south of Chile, using different attraction and restriction variables.

\begin{tabular}{|c|c|c|c|c|c|c|c|}
\hline $\begin{array}{l}\text { Variables } \\
\text { atracción/restricción }\end{array}$ & $\mathrm{Lk}^{1}$ & $\beta_{1}$ & $\beta_{2}$ & $\begin{array}{l}\beta_{3} \\
\%\end{array}$ & $\mathrm{R}_{2}$ & $\begin{array}{c}\mathrm{F} \\
\text { Snedecor }^{2}\end{array}$ & $\begin{array}{l}\text { Durbin- } \\
\text { Watson }^{3}\end{array}$ \\
\hline Población/distancia & $\begin{array}{c}3,065 \\
(1,719)\end{array}$ & $\begin{array}{l}-0,601 * \\
(-3,654)\end{array}$ & $\begin{array}{l}0,688^{*} \\
(6,072)\end{array}$ & $\begin{array}{l}-0,621^{*} \\
(-4,545)\end{array}$ & 38,0 & $14,506^{*}$ & 1,645 \\
\hline $\begin{array}{l}\text { Población/ } \\
\text { tiempo desplazamiento }\end{array}$ & $\begin{array}{c}3,074 \\
(1,730)\end{array}$ & $\begin{array}{l}-0,615^{*} \\
(-3,734)\end{array}$ & $\begin{array}{l}0,790 * \\
(6,366)\end{array}$ & $\begin{array}{l}-0,897 * \\
(-4,610)\end{array}$ & 38,4 & $\begin{array}{c}1 \\
4,755^{*}\end{array}$ & 1,555 \\
\hline $\begin{array}{l}\text { Ingreso/ } \\
\text { tiempo desplazamiento }\end{array}$ & $\begin{array}{l}-6,478 \\
(-1,048)\end{array}$ & $\begin{array}{l}-0,462 \\
(-1,037)\end{array}$ & $\begin{array}{l}1,301^{*} \\
(4,495)\end{array}$ & $\begin{array}{l}-0,485^{*} \\
(-2,530)\end{array}$ & 22,8 & $7,002 *$ & 1,352 \\
\hline $\begin{array}{l}\text { Patentes comerciales/ } \\
\text { tiempo desplazamiento }\end{array}$ & $\begin{array}{c}2,301 \\
(1,966)\end{array}$ & $\begin{array}{c}-0,211 \\
(-0,908)\end{array}$ & $\begin{array}{l}0,602 * \\
(3,937)\end{array}$ & $\begin{array}{l}-0,637 * \\
(-2,868)\end{array}$ & 27,7 & $5,733 *$ & 1,482 \\
\hline $\begin{array}{l}\text { Superficie comercial/ } \\
\text { tiempo desplazamiento }\end{array}$ & $\begin{array}{c}1,783 \\
(1,445)\end{array}$ & $\begin{array}{l}-0,152 \\
(-1,139)\end{array}$ & $\begin{array}{l}0,409 * \\
(3,875)\end{array}$ & $\begin{array}{l}-0,531^{*} \\
(-2,481)\end{array}$ & 26,0 & $5,266^{*}$ & 1,571 \\
\hline
\end{tabular}

${ }^{1}$ El valor Lk designa el logaritmo natural de la variable $\mathrm{k}$, y los valores $\beta 1, \beta 2$ y $\beta 3$ los coeficientes de las variables de cada especificación. Las cifras entre paréntesis corresponden al valor de $t$ de Student.

$2^{*}$, estadísticamente significativo ( $\left.\mathrm{p} \leq 0,05\right)$.

${ }^{3}$ Estadístico de Durban-Watson corresponde a una prueba para determinar la correlación serial entre los términos de error adyacentes (Vizauta, 2002).

${ }^{\prime}$ The $L k$ value designates the natural logarithm of the variable $k$, and the values $\beta 1, \beta 2$ y $\beta 3$ the coefficients of the variables of each specification. Numbers between parentheses correspond to the value of the tstudent.

$2^{*}$, statistically significant $(p<0.05)$.

${ }^{3}$ The Durban-Watson's test was used to estimate the serial correlation between adjacent error values (Vizauta, 2002). 
tamaño. Además, esto implica que la dimensión de la comuna actúa como factor de restricción de los flujos de gasto comercializable.

Se obtuvo coeficientes positivos correspondientes a la población, ingreso monetario, número de patentes comerciales y superficie de ventas de la comuna de destino de los flujos de gasto. Esto indica que el consumidor selecciona los centros de compra interurbanos de mayor dimensión como destino de sus desplazamientos de compra, utilizando el tamaño del centro comercial como indicador del atractivo del comercio. Al analizar en conjunto los signos de los coeficientes de las variables que caracterizan los centros evasores y receptores de gasto, se tiene que los flujos se dirigen desde las comunas de menor población hacia los centros más poblados, los cuales presentan mayores valores medios de compra dentro del entorno de residencia (Cuadro 1). El signo negativo de las variables de restricción utilizadas (distancia y tiempo de desplazamiento) indica que la distancia que recorre el consumidor para desplazarse a centros de compra interurbanos constituye un factor de restricción de los flujos de gasto.

Los coeficientes de las tres variables fueron significativos $(p \leq 0,05)$ en las especificaciones del modelo que utilizó la población como atributo de la comuna de origen y destino de los flujos comerciales, siendo levemente superior el coeficiente de determinación de la especificación que utilizó el tiempo de desplazamiento como variable de restricción. La bondad de ajuste del modelo no mejoró al introducir otras variables de atracción comercial de los centros de compra tales como el ingreso monetario de los hogares, número de patentes comerciales de establecimientos de venta de alimentos o superficie de ventas. Los coeficientes de la variable referida a la comuna desde la cual se evade gasto no fueron significativos $(p>0,05)$.

Respecto al ingreso monetario promedio de los hogares de origen de los flujos comerciales, se esperó una relación positiva y significativa entre éste y la intensidad de los flujos comerciales. No obstante, se obtuvo coeficiente de signo negativo y estadísticamente no significativo ( $p>0,05)$. Esto indica que el ingreso monetario de los consumidores dispuestos a desplazarse para realizar compras, no resulta determinante en las evasiones de gasto en la IX Región de Chile.

Luego de analizar los signos de los coeficientes de cada variable, se estudió la existencia de diferencias entre estos. Esto fue posible debido a que los coeficientes de cada una de las variables del modelo gravitacional tradicional, en su forma multiplicativa, fueron comparables entre sí, dado que los valores de los coeficientes correspondieron a las elasticidades (Pulido, 1987). De esta forma, considerando las características de la comuna de origen de los flujos de gasto se observó que las mayores elasticidades correspondieron a la población, - 0,601 para la especificación que usa la distancia y $-0,615$ en la que usa el tiempo de desplazamiento, seguida por el ingreso $(-0,462)$ en un nivel medio y por el número de licencias comerciales y superficie de venta $(-0,211$ y $-0,152$, respectivamente) (Cuadro 5). Según lo indica Medina (1997), fue posible esperar que parte de las evasiones de gasto que se producen puedan ser evitadas si las comunas alcanzan un determinado tamaño de población que rentabilice la actividad comercial.

Al analizar las diferencias existentes en los coeficientes de la variable tamaño de la comuna de destino, se obtuvo que la mayor elasticidad correspondió al ingreso mensual $(1,301)$, seguido por la población en la especificación que usa el tiempo de desplazamiento $(0,79)$; en un nivel intermedio, la población asociada a la distancia y el número de licencias comerciales (0,688 y 0,602 , respectivamente). La superficie comercial presentó una baja elasticidad. Una alta elasticidad respecto al tamaño de la comuna de destino, implica que para la compra de estos productos el consumidor se dirige específicamente hacia los centros de compra interurbanos de mayor dimensión. Sin embargo, los resultados obtenidos avalan que en forma paralela se privilegian los centros de compra con mayor poder adquisitivo. En relación a los resultados obtenidos para los coeficientes de la variable de restricción, es posible indicar que el tiempo de desplazamiento ejerce mayor efecto disuasivo en el desplazamiento de compra que la distancia a recorrer, con valores de elasticidad 
de $-0,897$ para el tiempo de desplazamiento y de -0,621 para la distancia. Mientras la elasticidad del tiempo de desplazamiento en la especificación que usa el número de patentes comerciales fue similar a la distancia, se obtuvo una elasticidad menor en las especificaciones que usan como variables de atracción el ingreso y la superficie comercial $(-0,485$ y $-0,531$, respectivamente). Esto implica que para la compra de alimentos, el consumidor está dispuesto a realizar desplazamientos de compra más largos hacia ciudades con mayor poder adquisitivo y superior superficie de venta.

Los coeficientes de la constante gravitacional, que indica el poder de atracción comercial existente entre dos centros comerciales en función de sus características (en este caso: población, ingreso, licencias comerciales y superficie de venta) y de la distancia que los separa, estadísticamente no fueron significativos $(\mathrm{p}<0,05)$ en las cinco especificaciones del modelo. Los coeficientes de determinación de las especificaciones (3) y (4) que utilizan la población como variables de atracción fueron mayores a $30 \%$, cifra considerada como aceptable para este tipo de estudios (Fotheringham y O'Kelly, 1989; Medina, 1997). Sin embargo, no fue así en las restantes especificaciones que tuvieron coeficientes de determinación inferiores. El supuesto de normalidad se cumplió en las cinco especificaciones, puesto que el histograma de residuos presentó una distribución que se aproxima a la normal y en el gráfico de probabilidad normal los residuos estandarizados presentaron distribución normal (siguen de cerca la diagonal) según indican Hair et al. (1997). Asimismo, los gráficos entre los residuos estandarizados y los valores pronosticados estandarizados permitieron comprobar el supuesto de homocedasticidad en las cinco especificaciones estimadas, al obtenerse una variabilidad de residuales relativamente constante a lo largo de los valores predichos (Visauta, 2002). El estadístico de Durbin-Watson no fue concluyente para probar o rechazar la hipótesis de autocorrelación en el término de error. Esto se debió a que se ubicaron en el intervalo de los valores dados por tabla según el número de observaciones de cada especificación y tres coeficientes estimados.

\section{Discusión}

En el área comercial de Temuco, la superficie de tiendas tradicionales representa 79,6\% de la superficie correspondiente a supermercados. Esto concuerda con la fuerte penetración de las cadenas de supermercados e hipermercados en la distribución al detalle en general, y en el sector alimentario en particular en Chile (Asociación Gremial de Supermercados de Chile, 2001). La mayor proporción de superficie concentrada en Temuco de ambos tipos de comercios de venta de alimentos, especialmente supermercados, explicaría que esta ciudad sea el primer destino elegido para comprar alimentos fuera del entorno de residencia, en la mayoría de las comunas que conforman esta área comercial (Cuadro 1). Es destacable el caso de las comunas de Pucón, Toltén, Collipulli, Lonquimay, Purén y Panguipulli, cuyos consumidores se desplazan más de 100 $\mathrm{km}$ para adquirir alimentos en Temuco. Esto se relaciona con la consideración de que cuanto mayor sea el tamaño de un establecimiento o la aglomeración de establecimientos, mayor variedad de surtido ofrecerá. Centros más grandes también ofrecen una mayor variedad de establecimientos comerciales donde elegir (LaForge et al., 1984; Medina, 1997). La variedad en el surtido se reconoce como uno de los factores fundamentales en la atracción de un destino de compras. En este sentido los estudios de Weisbrod et al. (1984) y Oppewal et al. (1997), concluyen que una atractiva mezcla comercial puede compensar desplazamientos más largos.

No obstante, es necesario señalar que las compras de alimentos por parte de consumidores de otras comunas en la ciudad de Temuco, probablemente se producen en el contexto de compras multipropósito. Es decir, con el fin de obtener ahorros de costo y tiempo. En el costo de la compra, los consumidores prefieren visitar establecimientos agrupados espacialmente a fin de adquirir diferentes bienes y servicios en un mismo desplazamiento (Medina, 1997; Dallaert y Arentze, 1998). Eppli y Benjamin (1994) afirman que, en la medida en que la mezcla comercial permita desplazamientos multipropósito, el consumidor estará dispuesto a recorrer distancias más largas. 
Por otra parte, se debe considerar el cambio en los hábitos de compra de productos de conveniencia como la alimentación. Existe una tendencia a una gran compra en reemplazo de una compra diaria (Nueno y Benett, 1997). Esta se realiza principalmente en supermercados e hipermercados elegidos por la variedad de productos, ofertas, descuentos y horarios de atención convenientes (Hernández et al., 1995). Esto concuerda con la mayor presencia de superficie comercial dedicada a supermercados en Temuco y, con los resultados obtenidos por Schnettler (2003), respecto a que los consumidores de $63 \%$ de las comunas del área comercial de Temuco eligen en primer lugar los supermercados, como formato comercial para comprar alimentos.

A partir de los resultados de la estimación del modelo de gravitación en la zona en estudio, fue posible validar el modelo para la compra de alimentos utilizando como variables explicativas la población de las comunas de origen, destino de los flujos de gasto y la distancia o el tiempo de desplazamiento. Los signos de los resultados coinciden con los obtenidos por Medina (1997) y por Gázquez y Sánchez (2000). Los coeficientes negativos de la variable tamaño de la comuna de origen indican la existencia de una relación inversa entre el nivel de población de la comuna de origen y el flujo de gasto intercambiable entre ellos. Esto implica que la dimensión de la comuna de origen actúa como factor de restricción de los flujos de gasto comercializable, concordando con estudios previos (Hawes y Lumpkin, 1984; Medina, 1997). Los coeficientes de la variable tamaño de la comuna de destino con signo positivo permiten indicar que cuanto mayor sea la población de la comuna de destino, mayor será el flujo de gasto que se intercambia con la comuna de origen. Esto concuerda con los resultados de LaForge et al. (1984), Mayo et al. (1988), Medina (1997), y O’Kelly (1999). Los coeficientes negativos de la variable distancia, indican que la distancia que tiene que recorrer el consumidor para desplazarse hacia centros de compra interurbanos, opera como factor de restricción de los flujos. Esto concuerda con los resultados obtenidos por Hortman et al. (1990), Marjanen (1997), Raijas (1997) y Orpana y Lampinen (2003) en relación con la adquisición de alimentos y por Fotheringham y O'Kelly (1989), Dennis et al. (2000), Arentze y Timmersmans (2001) en relación con la compra de otros productos.

La causa que explicaría que la bondad de ajuste del modelo no mejoró con la utilización de otras variables de atracción comercial; como el ingreso monetario de los hogares, el número de licencias comerciales o la superficie comercial correspondiente. Esto se podría explicar por puede radicar en el menor número de observaciones disponibles para la estimación del modelo, utilizando estas dos últimas variables. Dicha información sólo se pudo obtener en las comunas pertenecientes al área comercial de Temuco.

El ingreso de los consumidores de la IX Región de Chile no fue significativo para realizar desplazamientos de compra interurbanos para adquirir alimentos. La falta de significancia estadística de esta variable, junto al signo obtenido, permiten concluir la intensidad de los flujos de gasto de alimentos entre comunas no depende del ingreso de los consumidores de la IX Región. Estos resultados se contraponen a lo sugerido por Serrano (1990) respecto a la relación positiva entre el ingreso y los flujos de gasto evadidos. Del mismo modo, no concuerdan con lo determinado por Herrman y Beik (1968), Thompson (1971), Hawes y Lumpkin (1984), Bromley y Thomas (1995) y Sullivan y Savitt (1997) en el sentido que los consumidores con ingresos más altos realizan más compras fuera de la comuna de residencia. Esto en la IX Región se relaciona con la baja dotación comercial de venta de alimentos de las comunas de origen, en especial de supermercados (Cuadro 3), lo que obliga a los consumidores a desplazarse para proveerse de alimentos, independientemente del ingreso percibido.

Por tanto, independientemente de su renta, los consumidores de la IX Región de Chile realizan desplazamientos de compra fuera de la comuna de residencia para adquirir sus alimentos. Son atraídos por los centros de compra interurbanos de mayor población, que concentran los hogares de mayores ingresos y con mejor oferta comercial. Esto permite disminuir de los flujos 
de gasto, en la medida que se incrementa la distancia a recorrer.

\section{Resumen}

La desigual distribución del equipamiento comercial provoca desplazamientos de compra desde los centros de menor tamaño hacia los de mayor dotación comercial, provocando evasiones de gasto comercializable o flujos comerciales. Para determinar el efecto de variables de atracción y de restricción en los flujos comerciales de alimentos en las regiones VIII, IX y X de Chile, se estimó el modelo de gravitación tradicional. Los atributos de los centros de compra fueron población, ingreso monetario mensual de los hogares, cantidad de patentes y la superficie comercial de establecimientos de venta de alimentos. Como variable de restricción se utilizó la distancia y el tiempo de desplazamiento. Los consumidores que realizan desplazamientos de compra fuera de la comuna de residencia para adquirir alimentos seleccionan los centros de compra interurbanos de mayor población, que concentran los hogares de mayores ingresos, y cuya oferta comercial es superior, medida en número de comercios o tamaño de la superficie de ventas. El ingreso de los consumidores no es significativo para realizar desplazamientos de compra interurbanos, porque la baja dotación comercial en las comunas de origen obliga a los consumidores a desplazarse.

Palabras clave: áreas comerciales, desplazamientos de compra, gravitación comercial.

\section{Agradecimientos}

Los autores desean agradecer los aportes recibidos de la Fundación Ford, proyecto TEFE 150204, Dirección de Investigación y del Instituto de Desarrollo Local y Regional de la Universidad de La Frontera (UFRO); de la Dirección de Investigación,UFRO, proyecto DIUFRO 120601. Se agradece especialmente a Aldo Vergara Cubillos por su colaboración en la interpretación estadística de los resultados.

\section{Literatura citada}

ASACH. 2001. Página Web de la Asociación Gremial de Supermercados de Chile, www.asach.com (Consultado el 20 de marzo, 2002).

Arentze, T.A., and H.J.P. Timmersmans. 2001. Deriving performance indicators from models of multipurpose shopping behavior. Journal of Retailing and Consumer Services 8:325-334.

Bromley, R.D., and C.J. Thomas. 1995. Small town shopping decline: dependence and inconvenient for the disadvantaged. The International Review of Retail, Distribution and Consumer Research 5:433-456.

Casares, J. y A. Rebollo. 2000. Distribución Comercial. Editorial Civitas, Madrid, España. $267 \mathrm{pp}$.

Craig, C.S., A. Ghosh, and S. McLafferty. 1984. Models of the retail location process: a review. J. Retailing 60:5-36.

Dallaert, B., and T. Arentze. 1998. Investigating consumer's tendency to combine multiple shopping purposes and destinations. Journal of Marketing Research 35:177-188.

De Juan, M.D. 1998. La Atracción que Ejercen los Centros Comerciales Sobre los Consumidores. Servicio de Publicaciones de la Universidad de Alicante. Murcia, España. 139 pp.

Dennis, C.E., D. Marsland, and W.A. Cockett. 1999. Objects of desire: attraction and distance in shopping centre choice", Proceeding of the $10^{\text {th }}$ International Conference on Research of Distributive Trades, Institute for Retail Studies, Sterling. USA.

Eppli, M.J., and J.D. Benjamin. 1994. The evolution of shopping center research: a review and analysis. The Journal of Real Estate Research 9:5-31.

Fotheringham, A.S., and M.E. O'Kelly. 1989. Spatial Interaction Models: Formulation and Applications. Kluwer Academia Publishers, Dordrect, The Netherlands. $221 \mathrm{pp}$.

Gázquez, J. y M. Sánchez. 2000. Areas Comerciales, Capacidad de Compra y Riqueza en la Provincia de Almería. Monografías, Ciencias Económicas y Jurídicas. Almería, España. 122 pp.

Gil, I.A., A. Mollá y G. Berenguer. 1997. La imagen del punto de venta y su incidencia sobre la selección del establecimiento. AEDEMO 54: 46-54.

Hair, J., R. Anderson, R. Tatham y W. Black. 1999. Análisis Multivariante. Otero. Quinta ed. Prentice Hall Internacional. Inc. Madrid, España. 832 pp.

Hawes, J.M., and J.R. Lumpkin. 1984. Understanding the outshopper. Journal of Academy Marketing Science 12:200-218.

Hernández, M., J.L. Munuera y S. Ruiz de Maya. 1995. La estrategia de diferenciación en el comercio minorista. Información Comercial Española 739:27-45. 
Herrmann, R.O., and L. Beik. 1968. Shopper movements outside their local retail area. Journal of Marketing 32: 5-51.

Hortman, S., A. Allaway, B. Mason, and J. Rasp. 1990. Multi-segment analysis of supermarket patronage. Journal of Business Research 21:303-223.

Huff, D.L. 1964. Defining and estimating a trading area. Journal of Marketing 28:34-38.

INE. 2001. Quinta encuesta de presupuestos familiares (agosto 1996-julio 1997). Instituto Nacional de Estadísticas, Gobierno de Chile. Santiago, Chile. www.ine.cl/14-econo/ieconomia.htm. (Consultado el 17 de diciembre, 2001).

INE. 2003. Censo 2002. Resultados Volumen I: Población; País-Región. Instituto Nacional de Estadísticas, Gobierno de Chile. Santiago, Chile. 356 p.

LaForge, R., R. Reese, and W. Stanton. 1984. Identifying and attracting consumer outshoppers, Journal of Small Business Management, January 22-29.

Marjanen, H. 1997. Distance and Store Choice with Special Reference to Out-of-town Shopping, Ph.D. Dissertation, Department of Marketing, Turku School of Economic and Business Administration, Turku, Finland. 355 p.

Más, F. 1996. La imagen de los centros comerciales suburbanos en el modelo de Huff: una aplicación empírica, Libro de ponencias de AEDEM, 165178, Granada. España.

Matthews, R. 1992. Starving for food, few answers: supermarkets and hunger in America. Grocery Marketing 58:8-12.

Mayo, E.J., L. Jarvis, and J. A. Xander. 1988. Beyond the gravity model. Journal of the Academy of Marketing Science 16:23-29.

Medina, O. 1997. El Proceso de Selección de Centros de Compra Interurbanos por el Consumidor Español. Tesis Doctoral. Universidad Autónoma de Madrid, Facultad de Ciencias Económicas y Empresariales. Madrid, España. 373 p.

MIDEPLAN. 2000. Encuesta de caracterización socioeconómica nacional, CASEN 2000. Ministerio de Planificación Nacional, División Social, Departamento de Información Social, Gobierno de Chile, Santiago, Chile.
www.mideplan.cl/sitio/Sitio/casen/htm/casen encuesta.htm. (Consultado el 20 de marzo, 2004).

Nueno, J., and H. Bennett. 1997. The changing Spanish consume. International Journal of Research in Marketing 14:19-33.

O'Kelly, M. E. 1999. Trade-area models and choicebased samples. Environment and Planning A 1:613-627.

Oppewal, H., H. Timmermans, and J. Louviere. 1997. Modeling the effect of shopping centre size and store variety on consumer behavior. Environment and Planning A 29:1073-1090.

Orpana T., and J. Lampinen. 2003. Building spatial choice models from aggregate data. Journal of Regional Science 43:319-347.

Pulido, A. 1987. Modelos Econométricos. Ediciones Pirámide, Madrid, España. 447 p.

Raijas, A. 1997. The Consumer's Choice of Grocer's Shop-A Comparison Between two Metropolitan Areas in Finland and Norway. Ph.D. Dissertation, Faculty of Agriculture and Forestry, University of Helsinki, Helsinki, Finland. 338 p.

Scheaffer, R., W. Mendwenhall y L. Ott. 1996. Elementos de Muestreo. Grupo Editorial Iberoamericana S.A. Primera ed. Ciudad de México, México. 345 pp.

Schnettler, B. 2003. Atracción comercial ejercida por Temuco, IX Región de Chile, en productos de alimentación. Economía Agraria 8:365-375.

Serrano, F. 1990. Marketing para Economistas de Empresa. Editorial ESIS, Madrid, España. 223 pp.

Sullivan, P., and R. Savitt. 1997. Store patronage and lifestyle factors: implications for rural grocery retailers. International Journal of Retail and Distribution Management 25:351-364.

Thompson, J. R. 1971. Shopper mobility. Atlanta Economics Review 21:29-31.

Vizauta, B. 2002. Análisis Estadístico con SPSS para Windows. Vol I Estadística Básica. Mc Graw Hill. Segunda ed. Madrid, España. 332 pp.

Weisbrod, G.E., R.J. Parcells, and C. Kern. 1984. A disaggregate model for predicting shopping area market attraction. Journal of Retailing 60:65-83. 